\title{
The Story Behind the IFAS Assessment of Non-native Plants in Florida's Natural Areas ${ }^{1}$
}

\author{
A. M. Fox, D. R. Gordon, J. A. Dusky, L. Tyson, R. K. Stocker, K.A. Langeland, and A.L. Cooper²
}

\section{Introduction}

This document provides a history, justification, and summary of the IFAS Assessment of Non-Native Plants in Florida's Natural Areas (hereafter referred to as the IFAS Assessment).

The initial IFAS Assessment, now referred to as the Status Assessment, was developed in 1999 by a subcommittee of the IFAS Invasive Plants Working Group (IPWG) and consisted of a single component evaluating non-native plants that were known to be invading natural areas of Florida. Continued research efforts have helped to evolve and expand the IFAS Assessment into a well-defined mechanism that today is comprised of three components: the Status

Assessment, the Predictive Tool, and the Infraspecific Taxon Protocol. Documents pertaining to each of these three components are available to view and/or download from the IFAS Assessment of Non-Native Plants in Florida's Natural Areas website (http://plants.ifas.ufl.edu/assessment.html).
There is a growing awareness in Florida and nationally of problems related to non-native invasive species. For example, Wilcove et al. (1998) indicated that, in the United States, the problem of invasive species is second only to habitat loss as the leading threat to threatened and endangered species. Federal government recognition of the problems associated with invasive species was emphasized by President Clinton's Executive Order on Invasive Species, issued in 1999.

Only a small percentage of introduced species create problems in natural areas (Lippincott 1996), and most quantifiable ecological and economic impacts caused by the invasive plants in this group are negligible. However, a few invasive plant species have caused catastrophic damage.

At least two categories of invasive plants should be recognized: those currently in our wildland habitats and those that have not yet arrived. Ideally,

1. This document is SS-AGR-86, one of a series of the Agronomy Department, Florida Cooperative Extension Service, Institute of Food and Agricultural Sciences, University of Florida. First printed October 2000. Revised November 2009. It is an adaptation of an article first published in the Fall 2000 issue of Wildland Weeds, a quarterly publication of the Florida Exotic Pest Plant Council, Volume 3 (4): 4-7. Please visit the EDIS website at http://edis.ifas.ufl.edu.

2. A. M. Fox, Emeritus Associate Professor, Agronomy Department; D. R. Gordon, Senior Ecologist, The Nature Conservancy and Courtesy Professor, Department of Botany; J. A. Dusky, Professor and Associate Dean for Extension; L. Tyson, Professor, Santa Fe Community College, Gainesville, FL; R. K. Stocker, Emeritus Professor, Agronomy Department; K.A. Langeland, professor, Agronomy Department, Center for Aquatic and Invasive Plants; A.L. Cooper, biological scientist, Center for Aquatic and Invasive Plants; Florida Cooperative Extension Service, Institute of Food and Agricultural Sciences, University of Florida, Gainesville, FL 32611.

The use of trade names in this publication is solely for the purpose of providing specific information. UF/IFAS does not guarantee or warranty the products named, and references to them in this publication do not signify our approval to the exclusion of other products of suitable composition.

The Institute of Food and Agricultural Sciences (IFAS) is an Equal Opportunity Institution authorized to provide research, educational information and other services only to individuals and institutions that function with non-discrimination with respect to race, creed, color, religion, age, disability, sex, sexual orientation, marital status, national origin, political opinions or affiliations. U.S. Department of Agriculture, Cooperative Extension Service, University of Florida, IFAS, Florida A. \& M. University Cooperative Extension Program, and Boards of County Commissioners Cooperating. Millie Ferrer-Chancy, Interim Dean 
we could predict "invasion potential" of new species and prevent the introduction of new problems, or at least identify and eradicate them as soon as they are detected. Several predictive models are being developed (e.g., Australian Weed Risk Assessment: http://www.daff.gov.au/ba/reviews/weeds/system; Tucker and Richardson 1995; Parker et al. 2007), and many of them appear to be efficient at identifying potential problem species based on information such as whether a species has been invasive elsewhere.

A concern about many of these predictive models, however, has been that they are often overly restrictive, in some cases falsely accusing up to 20 30 percent of plants that have never (at least in the studied timescales) been found to be invasive (Reichard and Hamilton 1997, Gordon et al. 2008a). Managers of natural areas may not consider this excessive caution to be much of a flaw, but it is unacceptable to the many people who believe that supplies of plants for food, fiber, and landscaping should not be unnecessarily restricted.

If predictive models have their limitations, it seems that it should be easier to identify, describe, and assess invasive plants after they have escaped from cultivation and are appearing in natural areas. However, non-native plants are spread across a continuum of invasiveness that often changes over time. Also, "invasiveness" is a relatively subjective term; different people have varying perspectives on what constitutes minor versus significant invasive species impacts. It is not hard to recognize the extremes. The invasive "no-brainers" are typically well established and little disputed species, many of which are already subject to state and/or federal regulation (i.e., melaleuca - Melaleuca

quinquenervia, kudzu - Pueraria montana, cheatgrass - Bromus tectorum, etc.). On the other hand, many crop species of non-native origin with little or no known invasive potential require human intervention in the form of fertilizers, irrigation, etc. and therefore are not usually found in natural areas. Controversy, however, haunts the middle ground and usually surrounds those economically important species that are either just starting to escape into natural areas or that are already established in natural areas but with unknown or poorly documented impacts.

\section{Is Another Assessment Needed?}

Since 1984, the Florida Exotic Pest Plant Council (FLEPPC) has been classifying certain plants as Category I ("invasive exotics that are altering native plant communities" based "...on the documented ecological damage caused") or as Category II ("invasive exotics that have increased in abundance or frequency but have not yet altered Florida plant communities to the extent shown by Category I species"). These lists are revised biennially by a committee of experts within FLEPPC.

The lists serve a variety of purposes (see FLEPPC Invasive Plant Lists at http://www.fleppc.org/list/list.htm) with the objective to alert managers of natural areas to currently or potentially problematic species. Many natural areas within Florida are managed according to a policy that mandates removal and exclusion of all exotic plants. The FLEPPC lists help managers to prioritize invasive species for management, since few resource budgets allow for the removal of all exotic plants.

The issue becomes more controversial when the FLEPPC's lists are adopted for other purposes, such as the development of local ordinances banning the use of certain non-native plants. With a large gap between the FLEPPC lists and the state and federal regulations (on the 2007 lists, only 28 out of 67 Category I species and 7 out of 71 Category II species are government-regulated), it is not surprising that proactive local organizations have embraced the Category I list. Such regulations have alarmed ornamental horticulturalists and landscape designers, who question why some economically important species such as coral ardisia (Ardisia crenata), heavenly bamboo (Nandina domestica), and lantana (Lantana camara) are on the Category I list. Their concerns are magnified because although distribution information is available on the FLEPPC website, systematic written criteria and documented scientific evidence on which the FLEPPC lists are based are not available.

Conflicting opinions with regard to certain species have been mirrored within IFAS. Some IFAS faculty members recommend certain non-native 
species for landscaping while other faculty members support the FLEPPC lists and favor developing control programs for the same species. In an effort to resolve these conflicts within IFAS, a sub-committee of the IFAS Invasive Plants Working Group (IPWG) was established in early 1999 to develop a tool for assessing non-native plants in Florida's natural areas.

For many species already included on the FLEPPC Category I list, the IFAS Assessment has reached similar conclusions. For a few other species, however, the IFAS Assessment appears to express a reduced level of concern, and this is usually due to the IFAS Assessment's stringent criteria and requirements for documented evidence to show invasive potential.

The differences between the FLEPPC Category I list and the conclusions of the IFAS Assessment may seem alarming to some managers of natural areas. However, we anticipate that their alarm should provide the impetus to gather more evidence, especially for species with expanding ranges, so that problem species may be quickly recognized and reassessed. The precautionary approach of the FLEPPC lists is important for the managers of natural areas and should be continued. The IFAS Assessment is intended to complement FLEPPC's precautionary approach, and it is hoped that many people will contribute to the IFAS Assessment by providing information on their least-favorite plants.

\section{Purpose and Objectives of the IFAS Assessment}

The IFAS Assessment is comprised of three components: the Status Assessment (the initial component), the Predictive Tool, and the Infraspecific Taxon Protocol. The primary purpose of the IFAS Assessment is to provide a mechanism to be used within UF to develop consistent descriptions of, and recommendations for, the use and management of non-native plants in Florida. Secondary objectives are to: 1) include all the data that are available on any given species (such as in the FLEPPC or Florida Fish and Wildlife Conservation Commission (FWC) databases, or as reviewed by Langeland et al., 2008) so as to provide a level of information that is intermediate between simple presence or absence on a list and 2) identify the frequent data-gaps in our knowledge of these species, which would assist in setting research priorities. The IFAS Assessment could also be used as a tool to help resolve some of the conflicts identified by the liaison committee between FLEPPC and the Florida Nursery, Growers and Landscape Association (FNGLA).

The requirements for the IFAS Assessment are clear: it should have precisely defined criteria that are defendable by all UF/IFAS faculty, and all evidence and decisions should be documented and archived for anyone to review. Far less is published about most invasive species than desired for an assessment, and anecdotal information cannot be defended without substantiation. Thus, we have defined documentary evidence as being either published or as written observations from three biologists, any of whom could be contacted for confirmation. It is also important to recognize that the IFAS Assessment does not substitute for the FLEPPC lists, though some of the data may be useful for the FLEPPC list committee. Nor would this process be a sufficient replacement for formal (and much more costly and complex) risk-benefit analysis, such as is performed in the development of state regulations prohibiting the use of a species.

\section{General Overview of the Status Assessment}

After reviewing similar status assessments that have been developed elsewhere (e.g., Hiebert and Stubbendieck 1993), an early and important decision was made to limit the initial Status Assessment, as much as possible, to non-predictive information about existing plant populations in Florida. Predictive evaluations are necessary, particularly evaluations focusing on species not yet introduced to Florida, but the speculation inherent in prediction is different from the clear evidence approach of the Status Assessment. As a result, we decided to separate the two processes and incorporate the Predictive Tool into the IFAS Assessment. Additional lessons learned from other assessments were to provide quick exits from the evaluation for non-invasive species, to use multiple questions with simple choices (usually yes or no) but with mechanisms to acknowledge some uncertainty, and to uncouple a given species' level of 
impact from its current extent of invasion (so that an early invader is not automatically rated as of less concern than a widespread established species). We also decided to divide Florida into three zones (roughly corresponding to USDA growing zones) for which species would be assessed separately, a geographic distinction that was coincidentally incorporated into the 1999 FLEPPC lists. Typically, the IFAS Assessment is used at the species level, but where there are cultivars that differ in characteristics relevant to this assessment (e.g., cultivars, varieties, or sub-species), they should be evaluated separately using the newly added Infraspecific Taxon Protocol.

\section{Status Assessment}

The Status Assessment has five major sections: 1) define if a species is invading in Florida, 2) describe its ecological impacts, 3 ) identify its potential for expansion, 4) outline management difficulties, and 5) calculate its economic value. The focus of the Status Assessment is intentionally broader than just determining whether a species is invasive (e.g., the latter two indices provide important information unrelated to that issue), but the widening of the focus does not indicate an intention to offset economic value against ecological impacts.

An invading species is defined in Section I as one capable of establishing self-sustaining plant populations that are expanding within a natural plant community with which they had not previously been associated (Vitousek et al. 1995). To be declared as invasive, a plant must be documented in natural areas where there has not been significant human disturbance, or it must have survived restoration of the natural communities. Species invasiveness is evaluated within each of the three zones of the state (north, central, and south). A species that does not thus qualify as invading exits from the Status Assessment, unless it is known to hybridize with threatened, endangered, or economically important species.

Section II continues to evaluate each species separately for each zone, focusing in particular on the ecological impacts of the worst known site(s) of invasion, without or before any control effort. Scores are assigned in five categories that address disruption of ecosystem processes, impacts on threatened or endangered species, competitive displacement, changes in community structure, and hybridization with native species. The ecological impact score of a species is increased if it can invade a broad range of habitats. If the worst impacts are found in only a small proportion of all invaded sites, and if such sites can be defined and escape of a plant into those sites can be avoided, then limited uses of the plant may be specified to reduce the likelihood of such impacts occurring, but the "limited use" category is unlikely to apply to many species.

In zones that a plant has invaded, an assessment of high or low potential for further expansion (one of very few "predictive" questions) is based, in Section III, on the number of new sites reported to be infested in the last five years (using reports from the FLEPPC and/or FWC databases and other surveys). For zones where a species has not yet invaded, the potential for expansion is based on the likelihood that it could survive and cause impacts in the climate and habitats of that zone.

A species' difficulty of management and its economic value are assessed on a state-wide basis and result in scores based on 10 and 4 items, in Sections IV and V respectively. A species is considered more difficult to manage if non-target damage is hard to avoid, if access and methods of control are costly, if there are large or dispersed areas to be managed, or if the likelihood of re-growth and re-colonization is high. Economic value turned out to be the most challenging index because there is no method to track state-wide sales receipts by species. Nobody, including representatives from FNGLA, was very satisfied with the rather vague items in this section related to retail sales and importance to growers or farmers. Thus, an analysis of the economic impact of potentially invasive plants in the ornamental nursery industry has been proposed as an important area for future research.

\section{Predictive Tool}

In 2007 the Predictive Tool (revised in 2009) was added to the IFAS Assessment to evaluate species that are not present in Florida's natural areas. The Australian Weed Risk Assessment [WRA] (Pheloung et al., 1999) was adapted and tested for use in Florida (Gordon et al., 2008b) to complete the 
evaluation of such species. The Predictive Tool is applied to species that have not escaped into Florida's natural areas but are either recent arrivals to the state (less than 20 years if woody or less than 10 years if herbaceous) or are known to cause problems in areas with similar habitats and climate to Florida's habitats and climate, or if there is a proposed or new use for a species that would result in higher propagule pressure in Florida (e.g., cultivation of two or more contiguous acres of a species for bioenergy [corresponding to DPI biofuel rule], commercial cultivation of a species present in Florida for a new use, increase in acreage cultivated from 1-10 acres to 10 times that acreage [10-100 acres], 10-100 acres to 5 times that acreage [50-500 acres], or more than 100 acres to 2.5 times that acreage), then the Status Assessment directs the use of a predictive tool.

Similar to the Australian WRA, the Predictive Tool consists of 49 questions divided into three sections regarding the biogeography (section $\mathrm{A}$ ), the undesirable traits (section B) and the biology/ecology (section C) of the proposed plant species. A comprehensive literature review is performed to answer as many of the 49 questions as possible. However, to ensure that questions from each section are answered, a minimum of at least two questions in section $\mathrm{A}$, two questions in section $\mathrm{B}$, and six questions in section $\mathrm{C}$ are required. The answers are combined into a scoring system that determines the invasive potential of the proposed non-native plant species and ultimately concludes with a recommendation.

\section{Infraspecific Taxon Protocol}

The Infraspecific Taxon Protocol (hereafter referred to as the ITP) was added to the IFAS Assessment in 2008. It was designed to examine infraspecific taxa, such as cultivars, varieties, or sub-species, which are known to have different outcomes from the "resident species" (a.k.a. "parent species"). The ITP consists of three sections: Section 1) only applies to infraspecific taxa that can be distinguished in the field from the resident species, Section 2) only applies to infraspecific taxa that cannot be distinguished in the field from the resident species and for which the previously assessed resident species has a conclusion of "Caution; may be recommended but manage to prevent escape," "Invasive; not recommended," or "Predicted to be invasive; recommend only under specific management practices that have been approved by the IFAS Invasive Plants Working Group," and Section 3) zonal differences of the infraspecific taxon.

The ITP is initiated when a request to evaluate infraspecific taxa is submitted to the IFAS Assessment Team. The request must be supported by as much evidence as possible (i.e., publications, photographs, etc.) and include information about the date of the first introduction of the infraspecific taxon to Florida (or the United States if Florida data are not available); reasons for expecting the infraspecific taxon to behave differently and thus have a different conclusion from that of the resident species; and, if possible, the names and contact information of at least three qualified individuals (i.e., botanists, land managers, etc.) who are familiar with the taxon (this is necessary to answer question 1.1 with a "yes" response). If the evidence provided to the IFAS Assessment Team can be used to clearly answer Sections 2 and 3 and complete the assessment, then a draft of the results is provided to the requestor for an accuracy check before results are posted on the IFAS Assessment website. If, however, the request cannot be completed because of a lack of appropriate evidence, lack of three qualified individuals, or lack of agreement between the qualified individuals, then the infraspecific taxon is not listed separately from the resident species and it is assumed to have the same conclusions per zone as the resident species. If the requestor is not satisfied after the application of the ITP, any and all appeals must be addressed to the IFAS IPWG for a case-by-case review.

\section{IFAS Assessment Conclusions}

Authors of IFAS Extension publications discussing any of the species that have been assessed with this instrument are instructed to review, and where appropriate, use the language designated in the conclusions section (conclusions available at: http://plants.ifas.ufl.edu/assessment/conclusions.html ). While this language has no regulatory authority and is obviously superseded by any state or federal prohibitions, it is intended to provide consistent 
guidance to IFAS Extension personnel in making recommendations for use of these plants. It is important to remember that IFAS Extension programs provide information for our clientele, the end-users, whereas local, state, and federal agencies make regulatory decisions about what species can be planted and where. Whether or not the planting of that species should be permitted is an issue for the regulatory agencies.

\section{Status Assessment}

For all indices other than ecological impacts, the scores for a species are assigned to a high or low category. The combined scores for ecological impacts comprise the index that drives the development of conclusions. Ecological impact scores are assigned to low, medium, high, or very high categories. Based on the permutations of these high, low, etc. categories for each index, one of the following conclusions is designated by zone for a species:

- Not considered a problem species/infraspecific taxon; may be recommended (low impacts and potential for expansion);

- Caution: may be recommended, but manage to prevent escape (low impacts but high potential for expansion);

- Invasive: not recommended unless a specified and limited use has been approved by the IFAS Invasive Plants Working Group (medium to high impacts);

- Invasive: not recommended for any uses (high to very high impacts).

All species with a conclusion of not considered a problem species; may be recommended will be reassessed as new information becomes available (especially in relation to new sites or impacts) and at least every 10 years. Plants with caution or invasive and not recommended unless a specified and limited use has been approved by the IFAS Invasive Plants Working Group conclusions will be reassessed every two years.

For a few species with medium impacts and a conclusion of invasive and not recommended unless a specified and limited use has been approved by the
IFAS Invasive Plants Working Group, a caveat is included that if specific conditions for use could be defined from which escape and invasion could be prevented, then specific and limited-use recommendations could be proposed. Such proposals would have to be approved by the IFAS Invasive Plants Working Group.

Species that are rated with very high impacts, that score highly on all indices, or that have a combination of medium to high impacts, high potential and low value, are invasive and not recommended for use and will be reassessed as new information becomes available and at least every 10 years.

\section{Infraspecific Taxon Protocol}

The Infraspecific Taxon Protocol was developed so that the conclusion statements for infraspecific taxa would reflect those of the resident species from the Status Assessment. The only exception to this is if an infraspecific taxon would receive the conclusion Use of a predictive tool is recommended from the ITP response form. The Predictive Tool would then be applied to the infraspecific taxon separately. If, however, a lack of data or some other condition renders it impossible to apply the Predictive Tool to the infraspecific taxon, then the conclusion statement from the predictive tool for the resident species would be applied to the infraspecific taxa. Conclusions for infraspecific taxa that have been independently assessed using the ITP will be listed separately from the resident species in the conclusions table on the IFAS Assessment website.

\section{Predictive Tool}

Each of the 49 questions of the Predictive Tool is awarded a score between -3 and 5 points. The final point total leads to one of three outcomes: accept (less than 1 point), evaluate further (1-6 points), or reject (more than 6 points). Based on the outcome from the point total, one of the following conclusion statements is designated for a species (note: species currently listed within the conclusions table that have received a conclusion of "Not yet assessed" have either not yet been assessed, or were assessed before the induction of the Predictive Tool): 
- "Accept" = "Not a problem species, may be recommended" (reevaluate in 10 years using the Status Assessment, which may or may not direct the use of the WRA).

- "Evaluate further" = "Caution: may be recommended, but manage to prevent escape" (reevaluate in 2 years using the Status Assessment, which may or may not direct the use of the Weed Risk Assessment [WRA]. If the species has moved into natural area(s), retain the "Caution" conclusion for at least 10 years from the most recent predictive conclusion even if the Status Assessment conclusion is "not a problem species, may be recommended." The "Caution" conclusion must be retained in this instance because the species is clearly spreading in Florida. After ten years, adopt the conclusion suggested by the Status Assessment).

- "Reject" = "Predicted to be invasive: recommend only under specific management practices that have been approved by the IFAS Invasive Plant Working Group" (reevaluate in 2 years using the Status Assessment. If the species still requires a predictive assessment, update the WRA result with any new information. However, if the species has moved into natural area(s), retain the "Predicted to be invasive: not recommended" conclusion for woody species for 20 years and for herbaceous species for 10 years from the most recent predictive conclusion regardless of current status in natural area(s) because the species is clearly spreading in Florida).

This component is typically applied at the state level rather than to each zone of Florida. However, if results of a proposed non-native species would clearly vary across one or more of the zones because of an intolerance to an environmental condition (e.g., frost intolerance) or because of a constraining condition (e.g., a particular soil type found only in one zone), then the species would be assessed within each zone. Furthermore, if proposed non-native species result in an evaluate further outcome, the species are automatically re-evaluated by zone and further analyzed with the Pacific Second Screening developed by Daehler et al. (2004). The use of the
Pacific Second Screening helps to identify species as low- or high-risk pests of natural areas and agricultural (or other cultivated) lands.

\section{Perseverance of the IFAS Assessment}

Since the development of the IFAS Assessment in 1999, roughly 700 species have been tested with the formal collection of documentary evidence. These species represent all categories for each index and all conclusions for each component. Because data collection and documentation for each species requires a substantial effort, we have full- and part-time staff dedicated to this task (funding for this project is currently provided by the IFAS Office of the Dean for Research and the University of Florida Tropical and Subtropical Agricultural Trade and Policy Center T-STAR grant, and was previously funded by Florida DEP and FNGLA). Permanent funding for these positions would be advantageous to ensure continuous and consistent evaluation of non-native species.

The IFAS Assessment is an ever-changing mechanism that has become comprehensive over the last 10 years. For instance, the initial Status Assessment in 1999 was scrutinized within IFAS and by a number of external reviewers, resulting in revisions and approval for use by the IFAS Invasive Plants Working Group in 2001. Further revisions have been made over the last eight years, including some terminology changes $(2004,2005,2008$, and 2009) and the addition of the Predictive Tool and the Infraspecific Taxon Protocol in 2007 and 2008, respectively. We would like to have additional input on the IFAS Assessment itself and on the data that are collected for each species. We will also continue to test the structure and questions within the IFAS Assessment to see if there are redundant or overly influential questions, or to determine whether there are repeated data gaps. With a national growing awareness and concern about non-native invasive plants, other states, such as Ohio and Indiana, have shown interest in adapting this assessment for their local use (Fox et al. 2003). We expect that the IFAS Assessment will evolve continuously both from internal evaluations and from external input, hence the long-term objective of creating an interactive 
web-based version rather than just the printable format currently available.

\section{References}

Fox, A. M., D. R. Gordon and R. K. Stocker. 2003. Challenges of reaching consensus on assessing which non-native plants are invasive in natural areas. HortScience 38(1): 11-13.

Gordon, D.R., D.A. Onderdonk, A.M. Fox, and R.K. Stocker. 2008a. Accuracy of the Australian Weed Risk Assessment system across varied geographies. Diversity and Distributions 14: 234-242.

Gordon, D.R., D.A. Onderdonk, A.M. Fox, R.K. Stocker, and C. Gantz. 2008b. Predicting Invasive Plants in Florida Using the Australian Weed Risk Assessment. Invasive Plant Science and Management 1: $178-195$

Hiebert, R.D. and J. Stubbendieck. 1993. Handbook for ranking exotic plants for management and control. U.S. Dept. of Interior Natural Resources Report NPS/NRMWRO/NRR-93/08. Available online: http://nature.nps.gov/pubs/ranking/

Langeland, K.A., H.M. Cherry, C.M. McCormick, and K.A. Craddock Burks. 2008. Identification and Biology of Non-Native Plants in Florida's Natural Areas. $2^{\text {nd }}$ ed. Gainesville: IFAS Communications Services, University of Florida Publications. Publication SP 257. 193 p.

Lippincott, C. 1996. Current estimates of cultivated, native, naturalized, and weedy plant species in Florida. Florida Exotic Pest Plant Council Newsletter Summer 1996, Vol 6. No 3, p. 3.

Parker, C., Caton, B.P. and L. Fowler 2007. Ranking nonindigenous weed species by their potential to invade the United States. Weed Science 55: 386-397.

Pheloung, P.C, P.A. Williams, and S.R. Halloy. 1999. A weed risk assessment model for use as a biosecurity tool evaluating plant introductions. Journal of Environmental 57: 239-251.

Reichard, S.H. and C.W. Hamilton. 1997. Predicting invasions of woody plants introduced into North America. Conservation Biology 11:193-203.
Vitousek, P., L. Loope, C. D'Antonio and S.J. Hassol. 1995. Biological invasions as global change. pp. 213-336 In: S.J. Hassol and J. Katzenberger (eds) Elements of change 1994. Aspen Global Change Institute, Aspen, CO.

Tucker, K.C., and D.M. Richardson. 1995. An expert system for screening potentially invasive alien plants in South African fynbos. Journal of Environmental Management 44: 309-338.

Wilcove, D.S., D. Rothstein, J. Dubow, A. Phillips, and E. Losos. 1998. Quantifying threats to imperiled species in the United States. Bioscience 48:607-615. 